\title{
Precision measurements of rare kaon decays
}

\section{Evelina MARINOVA*i}

INFN Sezione di Perugia

E-mail: Evelina.Marinova@cern.ch

We report the recent results on rare kaon decays from the NA48/2 experiment. The precise measurement of direct photon emission (DE) in the decay $K^{ \pm} \rightarrow \pi^{ \pm} \pi^{0} \gamma$ and its interference (INT), with the INT amplitude being observed for the first time, has been finalized. This study is based on the full NA48/2 data set with about $600 \mathrm{k}$ reconstructed $K^{ \pm} \rightarrow \pi^{ \pm} \pi^{0} \gamma$ decays which is factor of 30 larger than for previous experiments. Samples of about 7200 reconstructed $K^{ \pm} \rightarrow \pi^{ \pm} e^{+} e^{-}$, and more than $3000 K^{ \pm} \rightarrow \pi^{ \pm} \mu^{+} \mu^{-}$events, with very small background contamination, have been collected. The latter is exceeding the total existing statistics by a factor of five. A precise measurement of the branching fractions and the form factors of the rare decays $K^{ \pm} \rightarrow \pi^{ \pm} e^{+} e^{-}$ has been performed using different theoretical models. The CP violating asymmetry between $\mathrm{K}^{+}$ and $K^{-}$in this channel is also measured.

European Physical Society Europhysics Conference on High Energy Physics, EPS-HEP 2009, July 16 - 222009

Krakow, Poland

\footnotetext{
${ }^{*}$ Speaker.

†n behalf of NA48/2 collaboration
} 
The NA48/2 experiment at the CERN-SPS, designed primarily for charge asymmetry measurements 「11, uses simultaneous $K^{+}$and $K^{-}$beams and has collected large samples of rare decays. Detailed information about the main detector and its performance can be found elsewhere [2].

$K^{ \pm} \rightarrow \pi^{ \pm} \pi^{0} \gamma$ decays

The properties of the $K^{ \pm} \rightarrow \pi^{ \pm} \pi^{0} \gamma$ decays are described in terms of the Lorentz invariant $W$ which is given by $W^{2}=\left(P_{K}^{*} \cdot P_{\gamma}^{*}\right)\left(P_{\pi}^{*} \cdot P_{\gamma}^{*}\right) /\left(m_{K} \cdot m_{\pi}\right)^{2}[3,4]$, where $P_{K}^{*}, P_{\gamma}^{*}, P_{\pi}^{*}$ are the particles momenta in the kaon rest frame. Both Inner bremsstrahlung (IB) and Direct emission (DE) contribute to the amplitude of the decay. DE can occur either through electric or through magnetic dipole transition. The electric dipole transition can interfere with the IB term giving rise to an interference term with $\mathrm{CP}$ violating contributions. The previous measurements of IB and DE, reported in reference [5], are performed in the region 55 to $90 \mathrm{MeV}$ in terms of kinetic energy of the pion in the kaon rest frame, $T_{\pi}^{*}$. The decay rate is written as

$$
\frac{d \Gamma^{ \pm}}{d W}=\frac{d \Gamma_{I B}^{ \pm}}{d W}\left[1+2 \cos \left( \pm \phi+\delta_{1}^{1}-\delta_{0}^{2}\right) m_{\pi}^{2} m_{K}^{2} X_{E} W^{2}+m_{\pi}^{4} m_{K}^{4}\left(\left|X_{E}\right|^{2}+\left|X_{M}\right|^{2}\right) W^{4}\right] .
$$

The NA48/2 measurement is made in an enlarged region of $T_{\pi}^{*},(0-80 \mathrm{MeV})$. After applying a cut on $W(0.2-0.9)$, about 600000$)$ events have been reconstructed, with a background estimation of about $1 \%$ of DE component (see Fig. 1 (a)). The mistagging probability for the radiated gamma is as low as one per mille. As the different contributions have a different $\mathrm{W}$ dependence, IB, INT and DE distributions can be well separated. The main technique used to extract INT and DE contributions is based on Poissonian Maximum Likelihood fit. The relative contribution of every source is calculated in such a way that the difference between the number of events and the total number of simulated events in every bin is minimal (see Fig. 1 (b), (c)). A cross-check was performed using a polynomial fit.
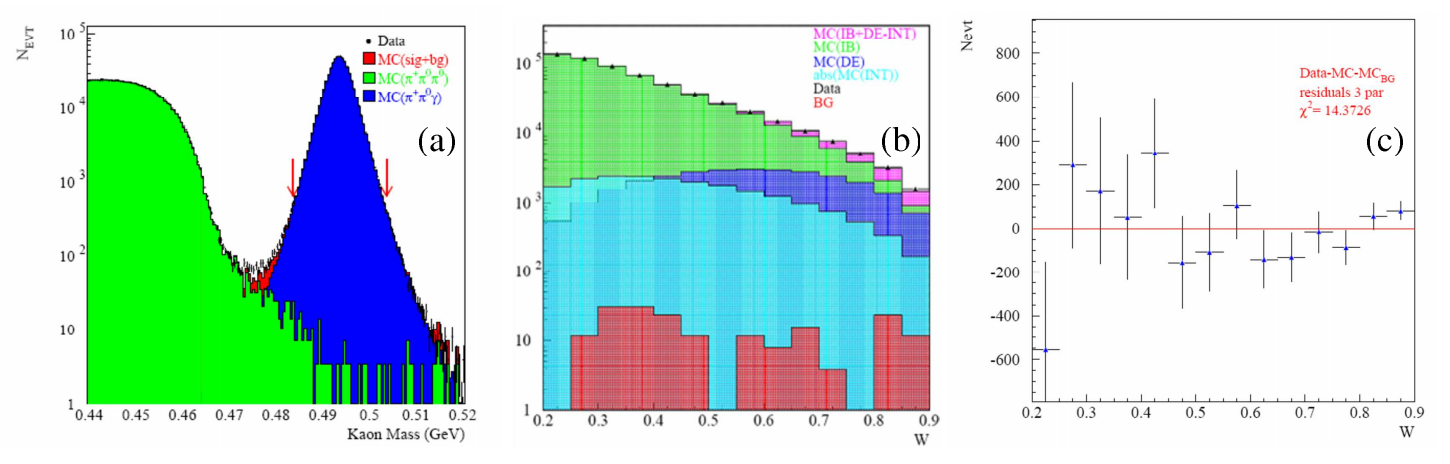

Figure 1: (a) Reconstructed mass of the kaon; (b) Contribution of the different components to the decay rate,their sum and the data distribution; (c) The fit residuals distribution.

The final results for the relative fraction of DE and INT with respect to IB are $\operatorname{Frac}(D E)_{T_{\pi}^{*}(0-80) \mathrm{MeV}}=$ $\left(3.32 \pm 0.15_{\text {stat }} \pm 0.14_{\text {sys }}\right) \cdot 10^{-2}, \operatorname{Frac}(I N T)_{T_{\pi}^{*}(0-80) \mathrm{MeV}}=\left(-2.35 \pm 0.35_{\text {stat }} \pm 0.39_{\text {sys }}\right) \cdot 10^{-2}$. In order to compare the result for DE, INT was assumed to be 0 , a polynomial fit was used, and $T_{\pi}^{*}$ is in the range $(55-90) \mathrm{MeV}$. The obtained result $B R(D E)_{T_{\pi}^{*}(55-90) \mathrm{MeV}}=\left(2.3 \pm 0.05_{\text {stat }} \pm 0.077_{\text {sys }}\right)$. $10^{-6}$ disagrees with the previous measurements [5] but the bad $\chi^{2}$ of the fit indicates that $I N T=0$ 
is a wrong assumption. The simultaneous measurement of INT and DE allows a measurement of the electric and the magnetic contributions to DE. The results are $X_{E}=\left(-24 \pm 4_{\text {stat }} \pm 4_{\text {sys }}\right) \mathrm{GeV}^{-4}$, $X_{M}=\left(254 \pm 11_{\text {stat }} \pm 11_{\text {sys }}\right) \mathrm{GeV}^{-4}$.

$K^{ \pm} \rightarrow \pi^{ \pm} e^{+} e^{-}$decays

The properties of the rare decays $K^{ \pm} \rightarrow \pi^{ \pm} \ell^{+} \ell^{-}$involving FCNC processes are described in terms of $z=M_{\ell \ell}^{2} / M_{K}^{2}$. The decay rate is given by $d \Gamma / d z \sim \rho(z) W(z)$ where $\rho(z)$ is a phase space factor. There are four theoretical models predicting the form factors $W(z)$ (see Tab. 1). Each model is characterized by two free parameters which determine a model dependent branching fraction. The measurements are done normalising to the $K^{ \pm} \rightarrow \pi^{ \pm} \pi_{\text {Dalitz }}^{0}$ which has the same charged particles in the final state. Due to the similarities between the signal and normalisation channel, systematics due to particle identification, trigger efficiencies, etc., is kept very low. In total 7253 events have been reconstructed with a background estimation of 1\% (see Fig. 2(a)). The kinematical region in $z$ is restricted to be larger than 0.8 (corresponding to $M_{e e}>140 \mathrm{MeV} / \mathrm{c}^{2}$ ) in order to suppress the background from the abundant normalisation channel. Fits to all models have been performed and have a reasonable quality (see Fig. 2(b).) The results are reported in Tab. 1. The combined result for a model dependent branching fraction is $B R=\left(3.11 \pm 0.04_{\text {stat }} \pm\right.$ $\left.0.05_{\text {syst }} \pm 0.08_{\text {ext }} \pm 0.07_{\text {model }}\right) \cdot 10^{-7}$ [9], in agreement with [10] and the predictions of [11]. A first

\begin{tabular}{c|c|c|c} 
Model & Form-factor & Parameter & Results with Combined error \\
\hline \multirow{3}{*}{ Model 1 } & $W(z)=G_{F} M_{K}^{2}\left|f_{0}\right|(1+\lambda z)$ & $\lambda$ & $2.32 \pm 0.18$ \\
& Linear & $\left|f_{0}\right|$ & $0.531 \pm 0.016$ \\
& & $B R_{1} \times 10^{-7}$ & $3.05 \pm 0.10$ \\
\hline \multirow{3}{*}{ Model 2 } & $W(z)=G_{F} M_{K}^{2} W_{+}^{\text {pol }}+W_{+}^{\pi \pi}(z)$ & $a_{+}$ & $-0.578 \pm 0.016$ \\
& ChPT at NLO [6] & $b_{+}$ & $-0.779 \pm 0.066$ \\
& & $B R_{2} \times 10^{-7}$ & $3.14 \pm 0.10$ \\
\hline \multirow{3}{*}{ Model 3 } & $W(\tilde{w}, \beta, z)$ & $\tilde{w}$ & $0.057 \pm 0.007$ \\
& Combined framework ChPT and & $\beta$ & $0.531 \pm 0.016$ \\
& Large- $\mathrm{N}_{c}$ QCD $|7|$ & $B R_{3} \times 10^{-7}$ & $3.13 \pm 0.10$ \\
\hline \multirow{3}{*}{ Model 4 } & $W(z) \equiv W\left(M_{a}, M_{\rho}, z\right)$ & $M_{a}$ & $0.974 \pm 0.035$ \\
& The ChPT parametrization involving the & $M_{\rho}$ & $0.716 \pm 0.014$ \\
& resonances $a$ and $\rho$ contribution $[8]$ & $B R_{4} \times 10^{-7}$ & $3.18 \pm 0.10$ \\
\hline n/a & restricted region in $M_{e e}>140 \mathrm{MeV} / \mathrm{c}^{2}$ & $B R_{m i} \times 10^{-7}$ & $2.28 \pm 0.08$
\end{tabular}

Table 1: Results of fits to the four models, and the model-independent branching ratio $B R_{m i}(z>0.08)$.

measurement of the direct $\mathrm{CP}$ violating asymmetry of $\mathrm{K}^{+}$and $\mathrm{K}^{-}$decay rates in the full kinematic range was obtained by performing BR measurements separately for $K^{+}$and $K^{-}$and neglecting the correlated uncertainties:

$\Delta\left(K_{\pi e e}^{ \pm}\right)=\left(B R^{+}-B R^{-}\right) /\left(B R^{+}+B R^{-}\right)=\left(-2.2 \pm 1.5_{\text {stat }} \pm 0.3_{\text {syst }}\right) \%$, where only the uncorrelated systematic errors have been taken into account. The result is compatible with no CP violation. However its precision is far from the theoretical SM expectation [6] of $\left|\Delta\left(K_{\pi e e}^{ \pm}\right)\right| \sim 10^{-5}$ and from the theoretical SUSY expectation of $\left|\Delta\left(K_{\pi e e}^{ \pm}\right)\right| \sim 10^{-3}[12]$. 


\section{$K^{ \pm} \rightarrow \pi^{ \pm} \mu^{+} \mu^{-}$decays}

About 310() events of the decay $K^{ \pm} \rightarrow \pi^{ \pm} \mu^{+} \mu^{-}$have been reconstructed from the full data sample. This sample is four times larger than the existing world statistics [5]. The main source of background is the abundant $K^{ \pm} \rightarrow 3 \pi^{ \pm}$decay (with subsequent $\pi \rightarrow \mu$ decay or with 2 misidentified $\pi$ ). The background is studied both with data and MC simulation and is at the order of few percent and well under control.
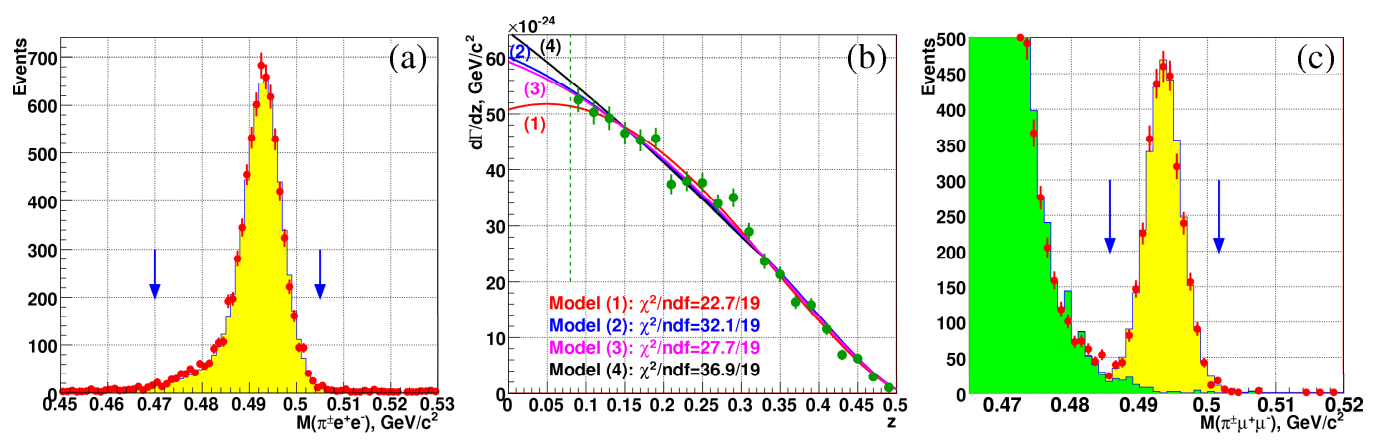

Figure 2: (a) The invariant mass of the kaon, reconstructed from $K^{ \pm} \rightarrow \pi^{ \pm} e^{+} e^{-}$decays; (b) The computed $(d \Gamma / d z)_{i}$ and the results of the $\chi^{2}$ of the fits corresponding to the considered models; (c) The invariant mass of the kaon, reconstructed from $K^{ \pm} \rightarrow \pi^{ \pm} \mu^{+} \mu^{-}$decays.

\section{Conclusions}

In conclusion, precise studies of several rare kaon decays measurements by the NA48/2 collaboration were presented. The achieved precisions are similar to or better than the best previous ones. Due to the simultaneous $K^{+}$and $K^{-}$beams, the CP violating charge asymmetry in $K^{ \pm} \rightarrow \pi^{ \pm} e^{+} e^{-}$was measured for the first time.

\section{References}

[1] J.R. Batley, et al., Eur. Phys. J. C 52 (2007) 875.

[2] V. Fanti, et al., Nucl. Instrum. Methods A 574 (2007) 433.

[3] N. Christ, Phys. Rev. 159, 1292 (1967).

[4] G. D'Ambrosio, M. Miragliuolo and P. Santorelli, The Daphne Physics Handbook, Eds. L. Maiani, G. Pancheri and N. Paver (1992).

[5] C. Amsler et al. [Particle Data Group], Phys. Lett. B 667, 1 (2008)

「6] G. D’Ambrosio, et al., JHEP 9808 (1998) 4.

[7] S. Friot, D. Greynat, E. de Rafael, Phys. Lett. B 595 (2004) 301.

「81 A.Z. Dubnickova, et al., Phys. Part. Nucl. Lett. 5 (2008) 76, hep-ph/0611175.

[9] J.R. Batley, et al., Phys. Lett. B 677 (2009), 246.

[10] P. Bloch, et al., Phys. Lett. B 56 (1975) 201. C. Alliegro, et al., Phys. Rev. Lett. 68 (1992) 278.

R. Appel, et al., Phys. Rev. Lett. 83 (1999) 4482.

[11] C. Bruno and J. Prades, Z. Phys. C57 (1993) 585; J. Prades, PoS(KAON)022, arXiv:0707.1789.

[12] A. Messina, Phys. Lett. B 538 (2002) 130.; G. D’Ambrosio, D.-N. Gao, JHEP 0207 (2002) 068. 